\title{
Management of Acute (Primary) Herpetic Gingivostomatitis in Immunocompetent Adult Patient: A Case Report
}

\author{
Andi Anggun Mauliana Putri \\ Oral Medicine Residency Program \\ Faculty of Dentistry, Universitas Indonesia \\ Jakarta, Indonesia \\ anggun.drg@gmail.com
}

\author{
Febrina Rahmayanti \\ Academic Staff of Oral Medicine Department \\ Faculty of Dentistry, Universitas Indonesia \\ Jakarta, Indonesia
}

\begin{abstract}
Acute (primary) herpetic gingivostomatitis (AHGS) is the most common form of HSV-1 infection in the oral cavity. These infections are reported mostly in children, usually localized and asymptomatic but in adult patients and immunocompromised conditions tend to be more severe, last longer, and can lead to systemic viremia. Case: A 46-year-old male patient came to RSGM FKG UI with a complaint of painful sore mouth, especially on the tongue since 5 days ago with previous history of fever. Patients feel pain around the head and while swallowing. On the examination, there were multiple ulcers at the gingival margin, interdental papillae, mucobuccofold, lateral of tongue, and also marginal gingivitis. Based on anamnesis, clinical and laboratory examination, he was diagnosed with AHGS. Case Management: He was prescribed chlorhexidine gluconate $0.2 \%$ mouthwash and multivitamin. He was advised to increase fluid intake, nutritious food, adequate rest to minimize the duration and the severity of symptoms. He was also instructed to improve the oral hygiene and encouraged to restrict direct contact to prevent transmission. Complete healing was seen in the next visit. Conclusion: Acute (primary) herpetic gingivostomatitis can be found in all age groups, including adult and elders. Clinical features of multiple ulcers and erythematous of gingival margins can be a pathognomonic sign of this condition. Primary HSV-1 infection usually manifests in the oral cavity so it is important for the dentist to recognize the disease to prevent misdiagnosis and provide appropriate management to the patient.
\end{abstract}

Keywords-acute (primary) herpetic gingivostomatitis, immunocompetent, adult

\section{INTRODUCTION}

About $60 \%$ - $95 \%$ of the population worldwide is infected by one or more viruses of viridae herpes family [1]. Acute (primary) herpetic gingivostomatitis (AHGS) is the most common clinical manifestation of herpes simplex virus infection that caused by herpes simplex type 1 virus (HSV-1 in $>90 \%$ of cases) or HSV-2 [2]. HSV-1 is primarily associated with skin and mucous membranes infection whereas type 2 is associated with genital infection, however the difference is more unclear [3]. Both forms of HSV have the same structure but different in antigenicity, although HSV-2 is known to have greater virulence. In addition, HSV-1 and HSV2 are neurotrophic alpha herpes viruses, has a fast replication cycle, and can infect anyone [4]. On the immunocompetent host, herpes viral infections often lead to debilitating disease [5]. Predisposing factor is a poor immune system, often accompanying such acute infectious conditions such as pneumonia, meningitis, influenza, typhoid, mononucleosis infections and stressful conditions. An infection transmitted through droplets and direct contact [6].

Acute (primary) herpetic gingivostomatitis (AHGS) typically affects children [7], but can affect people of any age [8]. Dentists have an advantage compared to other professionals in diagnosing the disease because of lesions in the oral cavity is the main symptom that affects the patient, therefore the dentist often become the first health practitioner to be visited by the patient [7]. Therefore, knowledge and early detection related to these diseases by the dentist is essential to prevent the spread of infection to other individuals [9].

In this paper, we report a case of acute (primary) herpetic gingivostomatitis in adult patients with immunocompetent conditions that expected to improve the knowledge of the dentist in recognizing the distinctive signs and symptoms of the AHGS condition that can also be found in adulthood in immunocompetent condition.

\section{CASE REPORT}

A 46-year-old man came to the Oral Medicine Department RSGM FKG UI with complaints there are sore stomatitis, especially on the tongue since 5 days ago. Two days before the onset of stomatitis, the patient had a fever and is still feeling the pain around the head and while swallowing at the present time so that the patient is difficult to eat and drink. Patients admitted in a state of exhaustion after going home. The fever has slightly subsides after the patient consume Cefixime ${ }^{\circledR}$ and Neuralgin ${ }^{\circledR}$ but stomatitis in the mouth, yet perceived increased and enlarged making him difficult to eat because he feels pain (VAS $=5$ ). The patient has been 5 days just consume milk, porridge, fruit juice and grass jelly. Pain also experienced when brushing teeth so the brushing frequency is reduced.

Patients have a history of recurrent stomatitis which usually occurs when he get a lot of office work. 
Stomatitis usually recovers quickly if the patient is taking a refreshing solution, however this time stomatitis are difficult to recover. This condition was never been experienced before. No history of systemic disease, allergies, family malignancy, smoking and alcohol consumption.

There is no rash on the body and a normal physical examination. On extraoral examination the submandibular glands and submental gland are palpable soft and painful. Lips look dry and there is desquamation. On intraoral examination, seen moderate oral hygiene; debris and stain; and there are subgingival and supragingival calculus. There are multiple, superficial, irregular edges ulcers, with a diameter of $1 \mathrm{~mm}$, white color-based ulcers on the marginal gingiva and interdental papillae, and there are ulceration in the mucobuccal fold in the 37-38 region; cheekbiting on right and left buccal mucosal. On the tongue there are also multiple ulceration, irregular edges, surrounded by erythematous halo, white ulcer base ulcers, sized $3 \times 2 \mathrm{~mm}$ in $42-43$ region and $2 \times 2 \mathrm{~mm}$ on 45-46 lateral tongue region, enlargement and erithematous on all linear gingival margins (Figure 1). It also seems thick white coating on the surface of the tongue and partial erupted tooth 48 .
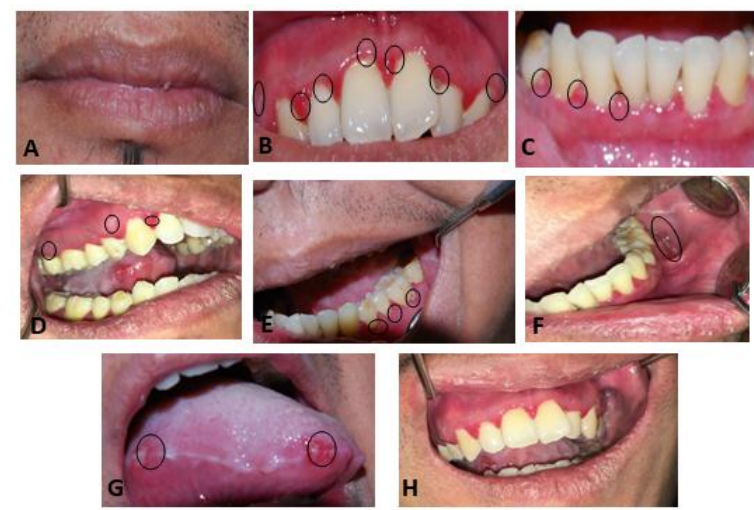

Figure 1. (A) Desquamation of the upper and lower lips. (B-F) Multiple ulcer on the marginal gingiva, interdental papillae, mucobuccal fold, and lateral tongue. $(\mathrm{H})$ enlargement and erithematous on all linear gingival margins.

On routine hematologic examinations show values within the normal range; IgM HSV-1 borderline whereas HSV-1 IgG, IgM HSV-2 and HSV-2 IgG negative (Table 1). Based on anamnesis, clinical and laboratory examination then concluded the diagnosis of this case is Acute (Primary) Herpetic gingivostomatitis (AHGS) with differential diagnosis Recurrent Intraoral Herpes (RIH) and stomatitis aphthous herpetiform.

\section{TABLE I. IMMUNOLOGICAL EXAMINATION OF HSV-1 AND} HSV-2 RESULTS

\begin{tabular}{|c|c|c|c|}
\hline $\begin{array}{c}\text { Examination } \\
\text { Types }\end{array}$ & Results & Reference Value & Unit \\
\hline IgG HSV-1 & Negative: 4.5 & Negative: $<20$ & \\
IgM HSV-1 & Borderline: 21.8 & U/mL \\
IgG HSV-2 & Negative: 5.5 & $\begin{array}{c}\text { Borderline: } 20-25 \\
\text { Positive: }>25\end{array}$ & \\
IgM HSV-2 & Negative: 15.2 & 25 & \\
\hline
\end{tabular}

\section{CASE MANAGEMENT}

Patients prescribed with Subex- $Z^{\circledR}$ tablets 1 time daily for 7 days and were advised to increase fluid and food intake with a soft-textured, balanced nutritional pattern, and enough rest to boost immunity. Patients are encouraged to improve oral hygiene by still brushing his teeth after breakfast and before bed and using a $0.2 \%$ chlorhexidine gluconate mouthwash twice daily. Patient also instructed to limit direct contact with people around to prevent transmission. In addition, patients are referred to the Periodontology Department to perform calculus cleansing that aims to eliminate the focus of infection. Patient is scheduled for reexamination 1 week later.

Patients come for control 2 weeks later, not according to the time that have been scheduled because he felt his condition was much better. On intraoral examination seen that the whole ulcers has healed and no new ulcer (Figure 2). Enlargement and the redness of the marginal gingiva also appears to be improving, but still there are supragingival and subgingival calculus, as well as debris and stain of the maxilla and mandible so that the patient is referred again to the Department of Periodontology.
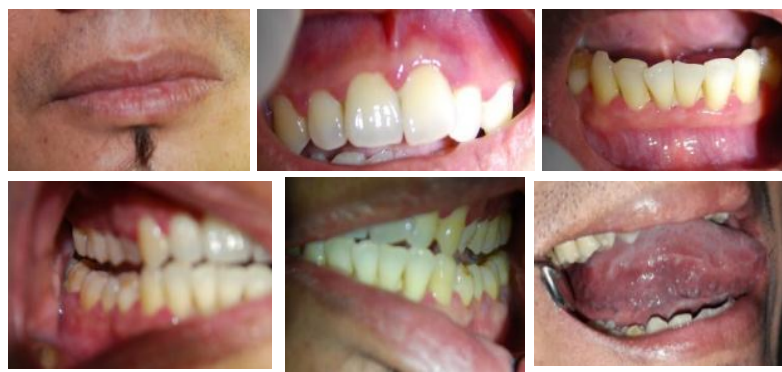

Figure 2. Clinical findings on second visits. Multiple ulcers had not been seen on the gingiva, mucobuccal fold and tongue and marginal gingivitis.

\section{DISCUSSION}

Acute Herpetic Gingivostomatitis (AHGS) generally occurs in childhood, between the ages of 6 months and 5 years old [7] that seen in the oral cavity before the age of 10 years old [10] and the second peak occurred in the early 20s [7]. Although onset is more common in children and young adults, primary infection occasionally is seen in much older people [10].

HSV viremia has been typically reported in immunocompromised hosts and neonatal HSV infections. However, with the advent of sensitive detection methods such as PCR, HSV viremia has been reported in $24 \%$ of immunocompetent subjects. Similarly, HSV DNA in the blood has been detected in $34 \%$ of children with primary herpetic gingivostomatitis and even in healthy patients with recurrences of herpes labialis [11].

Humans are the only natural reservoir of HSV infection and transmission can occur through direct contact with lesions or secretions from asymptomatic disease carriers. Latency, characteristic of all herpes viruses, occurs when the virus moves from the tip mucosal nerves or cutaneous through neurons to the 
ganglia where the HSV viral genome persists in a nonreplication phase. During the latent stage, herpes DNA can be detected, but it is not produce viral proteins. Reactivation of the latent virus occurs when HSV switches to a replicative state; this can occur as a result of a number of factors including peripheral tissue injury from trauma or sunburn, fever, or immunosuppression [4]; psychological stress; fatigue; menstruation; corticosteroid administration; nerve damage; and change in antiviral activity of the saliva [12].

Herpes can be transmitted in individuals that previously infected by the host which have symptomatic active lesions [10]. HSV had short-lived at the outer surface of the body so transmission of the virus must be through direct contact with infected lesions or body fluids, such as vesicle exudate, saliva, and genital fluid. In addition, the virus must be in contact with mucosa or skin who experience loss of integrity [7]. The severity of primary infection depends on the virus replication degree, the host response to foreign pathogens and the speed of the latency period [12].

AHGS is generally asymptomatic or accompanied by very mild symptoms that it goes unnoticed [6]. The incubation period is not fixed, from several days to 3 weeks, but in most cases it is approximately 1 week [7]. In symptomatic patient, prodromal non-pathognomonic signs and symptoms of fever, chills, malaise, irritability, headache, and anorexia usually occur after 2-20 days incubation period day [4]. The appearance of prodromal symptoms is thought to be caused by early virus replication on sensory nerve endings and in the epidermis or mucosa [1].

Within days of prodromal symptoms, erythema and groups of vesicles and/or ulcers can appear in all parts of the oral mucosa [7]. New vesicles continue to erupt for 3 up to 5 days, becoming rupture and fused within 24 to 48 hours results in shallow, irregular erosion or ulcers surrounded by red and painful halos. Healing occurs gradually, without scarring within 7 to 14 days, but HSV remains in saliva up to one month after the onset of the disease [4]. Gingiva usually appears bright red, swollen, and so painful that results in chewing difficulty [6]. Perioral skin may be involved due to infected salivary contamination [12].

Primary herpes simplex virus (HSV) infection on immunocompetent subjects usually involves one mucosal site although systemic and sometimes meningeal symptoms can be present [11]. In immunocompetent condition, the disease is self-limiting but resulting conditions can cause significant discomfort, burning around blisters, and eating and drinking difficulty [8]. Dehydration is a common complication of primary herpes which results in discomfort while eating and drinking [4].

The diagnosis of AHGS is usually based on clinical finding and history [2] that accurately assessed at $83.8 \%$ [3] so laboratory examination is rarely needed in this case [13]. However, it should be noted that the febrile, lymphadenopathic features are seen only in younger patients (patients aged 18 to 42 years), while older patients do not have lymphadenopathy [7]. In addition, adult patients may have a less common clinical finding and causing the diagnosis to be more complicated so sometimes necessary laboratory examination [4]. Diagnosis can be confirmed through laboratory tests, among other Serologic Tests (anti-HSV $\operatorname{IgM}$ and IgG), Tzanck test and immunofluorescence, but culture virus isolates are still considered as gold standard. In addition, HSV antibody testing can detect both types of virus at once, namely HSV-1 and HSV-2 [2].

Primary HSV infections are associated with increased $\operatorname{IgM}$ titers occurring in some days and followed by a continuous increase in $\mathrm{IgG}$ titers (seroconversion) several weeks later then indicating previously infection but not giving protection against reactivation [14]. The test results of newly infected individuals tend to show positive test results for herpes $\operatorname{IgM}$ and $\mathrm{IgG}$ or only herpes $\operatorname{IgM}$. IgM usually negative in recurrent HSV cases [2]. More specifically, high levels of $\operatorname{IgM}$ are consistent with a primary infection and acute levels of $\mathrm{IgG}$ are suggestive of a nonprimary infection [12]. In this case, the results of serological shows results of IgM and IgG HSV-1 were borderline whereas IgM and IgG HSV-1, and HSV-2 were negatives.

AHGS in adulthood usually benign and self-limiting [7] within 10-14 days [15] therefore treatment does not always given to patients with immunocompetent conditions [16], adjunctivemeasures may be undertaken to minimize the severity of symptoms [7]. However, additional therapies that are symptomatic and supportive can be given to minimize the duration and severity of symptoms [15]. Primary line treatments include the provision of nutritional supplements, encouraging patients to rest, avoiding the use of tobacco products and alcohol, the consumption of a balanced soft diet, and ensuring adequate fluid intake [4]. Systemic analgesic can be given to overcome the commonly accompanying pain and malaise [7].

The severity and quantity of intraoral lesions can reduce food intake significantly and cause patients to become dehydrated therefore it is important to offsetting the decrease in fluid intake that patients may experience. Nutritional supplements and soft foods diet can be recommended until the patient can tolerate solid foods [7]. In this case, patients are prescribed multivitamins and minerals that containing vitamins B1, B2, B3, B5, B6, B9, and B12; vitamin C, vitamin E, and zinc that aims to improve the immune system. However, nutritional supplements do not only improve the immune system, but also can accelerate the cure of herpes lesions. Vitamin C (Ascorbic acid) has been shown to inactivate a wide range of viruses in vitro, including Herpes simplex virus and to enhance immune function. Administering massive parenteral doses of vitamin $\mathrm{C}$ accelerated the healing of herpes lesions. Zinc ions have been shown to inhibit the replication of HSV-1 and -2 in vitro. A. It was suggested that this 
treatment be considered for prophylaxis prior to sun exposure for patients who experience sun-induced herpetic outbreaks. Topical application of vitamin E relieved pain and aided in the healing of oral herpetic lesions. Lithium can inhibited the replication of HSV-1 and HSV-2 in vitro at concentrations that did not inhibit host cell replication [4].

In addition to, the improvement of oral hygiene is also important. Though difficult to brush teeth, it is advisable to clean the plaque by brushing teeth paired with plaque control chemically using mouth rinse because dental hygiene and bad mouth can aggravate symptoms [17]. For oral infection, use of antiseptics such as chlorhexidine gluconate or a tetracycline rinse can lower secondary infections [15].

Provision of antiviral therapy (acyclovir) given 72 hours since the onset of symptoms [16] can accelerates the resolution of viral shedding and healing time, and reduces pain [2]. Aciclovir is active against herpes virses but does not eradicate them [15]. Early administration of antiviral therapy is very important because of the short duration of this disease. Ideally, treatment should begin before the lesion is apparent [1]. In these patients no oral acyclovir is administered due to viral replication and resolution which is characterized by prodromal symptoms, as the fever has occurred about 7 days ago as well the lesions are clearly visible in the oral cavity so that the administration of acyclovir is considered to be less effective.

As a conclusion, acute (primary) herpetic gingivostomatitis usually infect in childhood but can also be found in all age groups, including adult and elders. Clinical features of multiple ulcers and reddish gingival margins can be a distinctive sign of this condition. Diagnosis is generally based on clinical findings and symptoms, however laboratory examination sometimes required in adult patients because of the clinical features that appear may mimic recurrent HSV-1 infection conditions. This disease generally harmless and self-limiting in immunocompetent conditions so antivirus should not always be given.

Primary HSV-1 infection usually manifests in the oral cavity, so it is important for dentist to recognize this disease in order to prevent misdiagnosis and provide appropriate management to patients.

\section{ACKNOWLEDGMENT}

We would like to gratefully and sincerely thank drg. Siti Aliyah, Sp.PM as the clinical supervisor in this case.

\section{REFERENCES}

[1] A. Simmons, "Clinical manifestations and treatment considerations of herpes simplex virus infection,” J. Infect. Dis., vol. 186, pp. 71-77, 2002.

[2] A.K. George, S. Anil, "Acute herpetic gingivostomatitis associated with herpes simplex virus 2: report of a case," J. Int. Oral Health, vol. 6, pp. 99-102, 2014.

[3] E. Thomas, "A complication of primary herpetic gingivostomatitis,” Br. Dent. J., vol. 203(1), pp. 33-34, July 2007.

[4] S. Shah, P. Devi, Ravindra SV, K. Tyagi, D. Singh, "Primary herpetic gingivostomatitis: a case report and review of literature,” Tmu. J. Dent., vol. 1, pp. 119-123, July-Sept 2014.

[5] P.G. Arduino, S.R. Porter, "Herpes simplex virus type 1 infection: overview on relevant clinico-pathological features," J Oral Pathol. Med., vol. 37, pp. 107-121, 2008

[6] P. Jaya, K. Harijanti, "Gingivostomatitis herpetika primer (laporan kasus)," Oral Med. Dent. J., vol. 1, pp. 6-9, June December 2009.

[7] A.H. Ajar, P.J. Chauvin, "Acute herpetic gingivostomatitis in adults: a review of 13 cases, including diagnosis and management," J. Can. Dent. Assoc., vol.68, pp. 247-251, April 2002 .

[8] I.N. Staikov, N.V. Neykov, J.S. Kazandjieva, N.K. Tsankov, "Is herpes simplex a systemic disease?" Clin. Dermatol., vol. 33 pp. 551-555, 2015

[9] C.O. Cepeda, A.M. Valverde, M.M. Sabater-Recolons, E. JaneSalas, A. Mari-Roig, J. Lopez-Lopez, "A literature review and case report of hand, foot and mouth disease in an immunocompetent adult," BMC Res. Notes., vol. 9, pp. 165, 2016.

[10] N.S. Treister, M.A. Lerman, "Acute oral ulcerations," JADA. vol. 138, pp. 499-501, April 2007

[11] G. Boivin, B. Malette, N. Goyette, "Disseminated herpes simplex virus type 1 primary infection in a healthy individual,' Can. J. Infect. Dis. Med. Microbiol., vol. 20, pp. 122-124, 2009.

[12] M. Fatahzadeh, R.A. Schwartz, "Human herpes simplex virus infections: epidemiology, pathogenesis, symptomatology, diagnosis, and management," J. Am. Acad. Dermatol., vol. 57 pp. 1-3, November 2007.

[13] R.P.S. Mohan, S. Verma, U. Singh, N. Agarwal, "Acute primary herpetic gingivostomatitis,” BMJ. Case. Rep., pp. 1-3, 2013

[14] M. Glick, Burket's Oral Medicine, $12^{\text {th }}$ ed., USA: PMPH., 2015 , pp. 145-146.

[15] A. Field, T. Longman, Tyldesley's oral medicine, $5^{\text {th }}$ ed., New York: Oxford, 2004, pp. 40-44.

[16] S.S. Wilson, B.C. Herold, "Novel approaches in fighting herpes simplex virus infections," Expert Rev. Anti Infect. Ther., vol. 7 , pp. 559-568, June 2009.

[17] C. Jayam, A. Bandlapalli, K.R. Sharma, D. Chauhan, "Primary herpetic gingivostomatitis-detailed review and report of a case," J. Dent. Her., vol. 2, pp. 11-14, January 2015. 